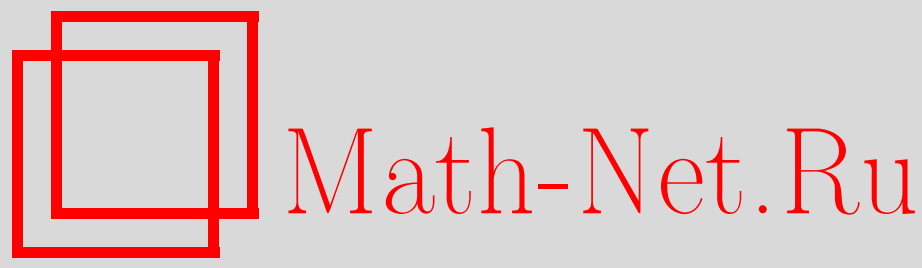

В. Д. Гордевский, А. А. Гукалов, Взаимодействие локально-максвелловских потоков в модели шероховатых сфер, ТМФ, 2013, том 176, номер 2, 322-336

DOI: https://doi.org/10.4213/tmf8457

Использование Общероссийского математического портала Math-Net.Ru подразумевает, что вы прочитали и согласны с пользовательским соглашением http://www . mathnet.ru/rus/agreement

Параметры загрузки:

IP: 52.6 .47 .48

26 апреля 2023 г., $12: 24: 32$

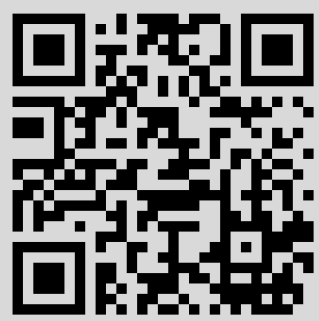


Том 176, № 2

август, 2013

\author{
$\begin{array}{ll}\text { (C) } 2013 \text { г. } & \text { В. Д. Гордевский* , А. А. Гукалов* }\end{array}$
}

\title{
ВЗАИМОДЕЙСТВИЕ ЛОКАЛЬНО-МАКСВЕЛЛОВСКИХ ПОТОКОВ В МОДЕЛИ ШЕРОХОВАТЫХ СФЕР
}

\begin{abstract}
Для нелинейного кинетического уравнения Бриана-Пиддака построены новые приближенные явные решения. Они имеют вид линейных комбинаций двух локальных максвеллианов типа "ускорение-уплотнение" и минимизируют равномерно-интегральную невязку с весом между частями уравнения.
\end{abstract}

Ключевые слова: уравнение Бриана-Пиддака, приближенные явные решения, "ускорение-уплотнение", невязка с весом.

DOI: $10.4213 / \operatorname{tmf} 8457$

\section{1. ВВЕДЕНИЕ}

В настоящей работе рассматривается модель шероховатых сфер [1]-[4], которую впервые ввел Бриан в 1894 г. [1]. Методы, развитые Чепменом и Энскогом для общих невращающихся сферических молекул, в 1922 г. были распространены Пиддаком [2] на модель Бриана. Преимущество этой модели перед всеми другими моделями, допускающими изменение состояния вращения молекул, состоит в том, что здесь не требуется никаких переменных, определяющих ориентацию молекулы в пространстве. Молекулы являются абсолютно упругими и абсолютно шероховатыми, что означает следующее. При столкновении двух молекул приходящие в соприкосновение точки в общем случае имеют разные скорости. Предполагается, что две сферы зацепляют друг друга без скольжения. В начальный момент сферы деформируют друг друга, а затем энергия деформации возвращается обратно в кинетическую энергию поступательного и вращательного движения без каких-либо потерь. В результате относительная скорость сфер в точке их соприкосновения изменяется при ударе на обратную.

Уравнение Больцмана для модели шероховатых сфер (или уравнение БрианаПиддака) имеет вид [3]-[6]

$$
D(f)=Q(f, f),
$$

* Харьковский национальный университет им. В. Н. Каразина, Харьков, Украина. E-mail: gordevskyy2006@yandex.ru,gukalex@ukr.net 


$$
\begin{gathered}
D(f) \equiv \frac{\partial f}{\partial t}+V \cdot \frac{\partial f}{\partial x} \\
Q(f, f) \equiv \\
\quad \frac{d^{2}}{2} \int_{\mathbb{R}^{3}} d V_{1} \int_{\mathbb{R}^{3}} d \omega_{1} \int_{\Sigma} d \alpha B\left(V-V_{1}, \alpha\right) \times \\
\times\left[f\left(t, V_{1}^{*}, x, \omega_{1}^{*}\right) f\left(t, V^{*}, x, \omega^{*}\right)-f(t, V, x, \omega) f\left(t, V_{1}, x, \omega_{1}\right)\right] .
\end{gathered}
$$

Здесь $d$ - диаметр молекулы, который связан с моментом инерции $I$ следующим соотношением:

$$
I=\frac{b d^{2}}{4}
$$

где $b \in(0,2 / 3]$ - параметр, характеризующий изотропное распределение вещества внутри частицы газа, $t$ - время, $x=\left(x^{1}, x^{2}, x^{3}\right) \in \mathbb{R}^{3}$ - пространственная координата, $V=\left(V^{1}, V^{2}, V^{3}\right)$ и $w=\left(w^{1}, w^{2}, w^{3}\right) \in \mathbb{R}^{3}$ - линейная и угловая скорости молекулы соответственно, $\partial f / \partial x$ - градиент функции $f$ по переменной $x$ (в дальнейшем будем также коротко обозначать его через $\left.f^{\prime}\right), \Sigma$ - единичная сфера в пространстве $\mathbb{R}^{3}$, $\alpha$ - единичный вектор из $\mathbb{R}^{3}$, направленный вдоль линии, соединяющей центры сталкивающихся молекул,

$$
B\left(V-V_{1}, \alpha\right)=\left|\left(V-V_{1}, \alpha\right)\right|-\left(V-V_{1}, \alpha\right)
$$

- столкновительный член.

Линейные $\left(V^{*}, V_{1}^{*}\right)$ и угловые $\left(w^{*}, w_{1}^{*}\right)$ скорости молекул после столкновения выражаются через соответствующие скорости до столкновения следующим образом:

$$
\begin{aligned}
& V^{*}=V-\frac{1}{b+1}\left(b\left(V_{1}-V\right)-\frac{b d}{2} \alpha \times\left(\omega+\omega_{1}\right)+\alpha\left(\alpha, V_{1}-V\right)\right), \\
& V_{1}^{*}=V_{1}+\frac{1}{b+1}\left(b\left(V_{1}-V\right)-\frac{b d}{2} \alpha \times\left(\omega+\omega_{1}\right)+\alpha\left(\alpha, V_{1}-V\right)\right), \\
& \omega^{*}=\omega+\frac{2}{d(b+1)}\left\{\alpha \times\left(V-V_{1}\right)+\frac{d}{2}\left[\alpha\left(\omega+\omega_{1}, \alpha\right)-\omega-\omega_{1}\right]\right\}, \\
& \omega_{1}^{*}=\omega_{1}+\frac{2}{d(b+1)}\left\{\alpha \times\left(V-V_{1}\right)+\frac{d}{2}\left[\alpha\left(\omega+\omega_{1}, \alpha\right)-\omega-\omega_{1}\right]\right\} .
\end{aligned}
$$

Общий вид максвелловского распределения для рассматриваемой модели был найден в работе [6]. В частности, там был получен явный вид максвелловского распределения, описывающего движение типа "ускорение-уплотнение" газа для этой модели. Нашей задачей является изучение процесса взаимодействия двух потоков указанного типа.

Будем использовать следующую невязку, впервые предложенную в работе [7] (равномерно-интегральная или "смешанная" невязка с весом):

$$
\widetilde{\Delta}=\sup _{(t, x) \in \mathbb{R}^{4}} \frac{1}{1+|t|} \int_{\mathbb{R}^{3}} d V \int_{\mathbb{R}^{3}} d \omega|D(f)-Q(f, f)| .
$$

Рассмотрим бимодальное распределение

$$
f=\varphi_{1} M_{1}+\varphi_{2} M_{2},
$$


где $\varphi_{i}=\varphi_{i}(t, x)$ (индекс $i$ здесь и всюду ниже принимает значения 1 и 2 ), а максвеллианы $M_{i}$ соответствуют "ускоряющемуся-уплотняющемуся" движению и имеют следующий вид [6], [8]:

$$
M_{i}=\rho_{i} I^{3 / 2}\left(\frac{\beta_{i}}{\pi}\right)^{3} e^{-\beta_{i}\left(\left(V-\bar{V}_{i}\right)^{2}+I \omega^{2}\right)},
$$

где $\rho_{i}-$ плотность газа:

$$
\rho_{i}=\rho_{0 i} e^{\beta_{i}\left(\bar{V}_{i}^{2}+2 \bar{u}_{i} x\right)},
$$

a

$$
\bar{V}_{i}=\widehat{V}_{i}-\bar{u}_{i} t
$$

- массовая скорость, $\beta_{i}=1 / 2 T$ - обратная температура, $\rho_{0 i}, \bar{u}_{i}, \widehat{V}_{i}$ - произвольные постоянные из пространств $\mathbb{R}$ и $\mathbb{R}^{3}$.

В разделе 2 получены результаты, приводящие к достаточным условиям минимизации невязки (7) за счет подходящего выбора коэффициентных функций $\varphi_{i}$ и параметров распределения.

\section{2. ОСНОВНЫЕ РЕЗУЛЬТАТЫ}

TEOPEMA 1. Пусть

$$
\varphi_{i}(t, x)=\psi_{i}(t, x) e^{-2 \beta_{i} \bar{u}_{i} x},
$$

где функиии $\psi_{i}$ таковы, что произведения множителя

$$
e^{\beta_{i}\left(\widehat{V}_{i}-\bar{u}_{i} t\right)^{2}} \frac{1}{1+|t|}
$$

на величины

$$
\psi_{i}, \quad \frac{\partial \psi_{i}}{\partial t}, \quad\left|\frac{\partial \psi_{i}}{\partial x}\right|, \quad \psi_{i} t, \quad t\left(\bar{u}_{i}, \frac{\partial \psi_{i}}{\partial x}\right)
$$

ограничены по $t, x$ на $\mathbb{R}^{4}$. Пусть, кроме того, выполняются соотношения

$$
\begin{aligned}
& \bar{u}_{i}=\frac{s_{i} \bar{u}_{0 i}}{\sqrt{\beta_{i}}}, \\
& \widehat{V}_{i}=\frac{s_{i} \widehat{V}_{0 i}}{\beta_{i}^{k_{i}}},
\end{aligned}
$$

где $\bar{u}_{0 i}, \widehat{V}_{0 i} \in \mathbb{R}^{3}$ - некоторые фиксированные векторы, $s_{i}>0$ - произвольная константа, а показатели степени $k_{i}$ удовлетворяют неравенству

$$
k_{i} \geqslant \frac{1}{2}
$$

Тогда существует такая величина $\widetilde{\Delta}^{\prime}$, что

$$
\widetilde{\Delta} \leqslant \widetilde{\Delta}^{\prime}
$$

причем она имеет следующие конечные пределы: 
а) при $k_{i}>1 / 2$ верно равенство

$$
\begin{aligned}
& \lim _{\beta_{i} \rightarrow+\infty} \widetilde{\Delta}^{\prime}=\sum_{i=1}^{2} \rho_{0 i} \sup _{(t, x) \in \mathbb{R}^{4}}\left\{\frac{1}{1+|t|} e^{t^{2} s_{i}^{2} \bar{u}_{0 i}^{2}}\left|\frac{\partial \psi_{i}}{\partial t}+2 \psi_{i} t s_{i}^{2} \bar{u}_{0 i}^{2}\right|\right\}+ \\
& +\frac{4}{\sqrt{\pi}} \sum_{i=1}^{2} s_{i} \rho_{0 i}\left|\bar{u}_{0 i}\right| \sup _{(t, x) \in \mathbb{R}^{4}}\left\{\frac{1}{1+|t|} e^{t^{2} s_{i}^{2} \bar{u}_{0 i}^{2}} \psi_{i}\right\}
\end{aligned}
$$

б) при $k_{i}=1 / 2$ получаем

$$
\begin{aligned}
\lim _{\beta_{i} \rightarrow+\infty} \widetilde{\Delta}^{\prime}= & \sum_{i=1}^{2} \rho_{0 i} \sup _{(t, x) \in \mathbb{R}^{4}}\left\{\frac{1}{1+|t|}\left|\frac{\partial \psi_{i}}{\partial t}+2 \psi_{i} t s_{i}^{2} \bar{u}_{0 i}^{2}\right| e^{s_{i}^{2}\left(\widehat{V}_{0 i}-\bar{u}_{0 i} t\right)^{2}}\right\}+ \\
& +2 \sum_{i=1}^{2} \rho_{0 i}\left(\frac{2 s_{i}\left|\bar{u}_{0 i}\right|}{\sqrt{\pi}}+s_{i}^{2}\left(\bar{u}_{0 i}, \widehat{V}_{0 i}\right)\right) \sup _{(t, x) \in \mathbb{R}^{4}}\left\{\frac{1}{1+|t|} e^{s_{i}^{2}\left(\widehat{V}_{0 i}-\bar{u}_{0 i} t\right)^{2}} \psi_{i}\right\} .
\end{aligned}
$$

ДокАзАтельство. Прежде чем сделать оценку сверху для невязки (7), мы оценим подынтегральное выражение (7). Используя формулы (2), (3) и (8) и с учетом того, что максвеллианы $M_{i}$ обращают в нуль обе части уравнения (1), можно получить соотношения

$$
\begin{gathered}
D(f)=M_{1} D\left(\varphi_{1}\right)+M_{2} D\left(\varphi_{2}\right)=M_{1}\left(\frac{\partial \varphi_{1}}{\partial t}+V \frac{\partial \varphi_{1}}{\partial x}\right)+M_{2}\left(\frac{\partial \varphi_{2}}{\partial t}+V \frac{\partial \varphi_{2}}{\partial x}\right) \\
Q(f, f)=\varphi_{1} \varphi_{2}\left[Q\left(M_{1}, M_{2}\right)+Q\left(M_{2}, M_{1}\right)\right] .
\end{gathered}
$$

Используем следующее разложение интеграла столкновений [9]:

$$
Q(f, g)=G(f, g)-f L(g)
$$

где уменьшаемое называют прибыточным членом:

$$
G(f, g)=\frac{d^{2}}{2} \int_{\mathbb{R}^{3}} d V_{1} \int_{\mathbb{R}^{3}} d \omega_{1} \int_{\sum} d \alpha B\left(V-V_{1}, \alpha\right) f\left(t, x, V_{1}^{*}, \omega_{1}^{*}\right) g\left(t, x, V^{*}, \omega^{*}\right)
$$

a $L(g)$ - затратный член интеграла столкновений (3), который допускает представление

$$
L(g)=\frac{d^{2}}{2} \int_{\mathbb{R}^{3}} d V_{1} \int_{\mathbb{R}^{3}} d \omega_{1} \int_{\sum} d \alpha B\left(V-V_{1}, \alpha\right) g\left(t, x, V_{1}, \omega_{1}\right) .
$$

В работе [10] было показано, что

$$
\int_{\mathbb{R}^{3}} d V \int_{\mathbb{R}^{3}} d \omega Q\left(M_{i}, M_{j}\right)=0
$$


а это согласно разложению (22) означает, что

$$
\int_{\mathbb{R}^{3}} d V \int_{\mathbb{R}^{3}} d \omega G\left(M_{i}, M_{j}\right)=\int_{\mathbb{R}^{3}} d V \int_{\mathbb{R}^{3}} d \omega M_{i} L\left(M_{j}\right) .
$$

Тогда верно следующее неравенство:

$$
\begin{aligned}
|D(f)-Q(f, f)| \leqslant & M_{1}\left(\left|D\left(\varphi_{1}\right)\right|+\varphi_{1} \varphi_{2} L\left(M_{2}\right)\right)+M_{2}\left(\left|D\left(\varphi_{2}\right)\right|+\varphi_{1} \varphi_{2} L\left(M_{1}\right)\right)+ \\
& +\varphi_{1} \varphi_{2}\left(G\left(M_{1}, M_{2}\right)+G\left(M_{2}, M_{1}\right)\right) .
\end{aligned}
$$

Интегрируя последнее неравенство по всему пространству линейных и угловых скоростей и используя равенство (26), получим

$$
\begin{aligned}
\int_{\mathbb{R}^{3}} d V \int_{\mathbb{R}^{3}} d \omega|D(f)-Q(f, f)| \leqslant & \sum_{i=1}^{2} \int_{\mathbb{R}^{3}} d V \int_{\mathbb{R}^{3}} d \omega M_{i}\left|D\left(\varphi_{i}\right)\right|+ \\
& +4 \varphi_{1} \varphi_{2} \int_{\mathbb{R}^{3}} d V \int_{\mathbb{R}^{3}} d \omega G\left(M_{1}, M_{2}\right) .
\end{aligned}
$$

Используем формулу [5]

$$
\int_{\mathbb{R}^{3}} d V \int_{\mathbb{R}^{3}} d \omega G\left(M_{1}, M_{2}\right)=\frac{d^{2} \rho_{1} \rho_{2}}{\pi^{2}} \int_{\mathbb{R}^{3}} \int_{\mathbb{R}^{3}} d q d q_{1} e^{-q^{2}-q_{1}^{2}}\left|\frac{q}{\sqrt{\beta_{1}}}-\frac{q_{1}}{\sqrt{\beta_{2}}}+\bar{V}_{1}-\bar{V}_{2}\right|
$$

а именно подставим вместо интеграла от прибыточного члена в выражение (28) правую часть равенства (29) и учтем (11):

$$
\begin{aligned}
\int_{\mathbb{R}^{3}} d V \int_{\mathbb{R}^{3}} d \omega|D(f)-Q(f, f)| \leqslant & \sum_{i=1}^{2} \int_{\mathbb{R}^{3}} d V \int_{\mathbb{R}^{3}} d \omega\left|D\left(\varphi_{i}\right)\right| M_{i}+ \\
& +\frac{4 \varphi_{1} \varphi_{2} d^{2} \rho_{1} \rho_{2}}{\pi^{2}} \int_{\mathbb{R}^{3}} \int_{\mathbb{R}^{3}} d q d q_{1} e^{-q^{2}-q_{1}^{2}} \Theta
\end{aligned}
$$

где

$$
\Theta=\left|\frac{q}{\sqrt{\beta_{1}}}-\frac{q_{1}}{\sqrt{\beta_{2}}}+\widehat{V}_{1}-\widehat{V}_{2}+\left(\bar{u}_{2}-\bar{u}_{1}\right) t\right| .
$$

Далее воспользуемся следующим очевидным равенством:

$$
\int_{\mathbb{R}^{3}} d \omega e^{-\beta_{i} I \omega^{2}}=\left(\frac{\pi}{\beta_{i} I}\right)^{3 / 2}
$$

тогда правая часть неравенства (30) примет вид

$\rho_{i}\left(\frac{\beta_{i}}{\pi}\right)^{3 / 2} \sum_{i=1}^{2} \int_{\mathbb{R}^{3}} d V\left|\frac{\partial \varphi_{i}}{\partial t}+V \frac{\partial \varphi_{i}}{\partial x}\right| e^{-\beta_{i}\left(V-\bar{V}_{i}\right)^{2}}+\frac{4 \varphi_{1} \varphi_{2} d^{2} \rho_{1} \rho_{2}}{\pi^{2}} \int_{\mathbb{R}^{3}} \int_{\mathbb{R}^{3}} d q d q_{1} e^{-q^{2}-q_{1}^{2}} \Theta$ 
после чего, осуществляя замену переменных $p^{2}=\beta_{i}\left(V-\bar{V}_{i}\right)^{2}$, получаем

$$
\begin{aligned}
\int_{\mathbb{R}^{3}} d V \int_{\mathbb{R}^{3}} d \omega|D(f)-Q(f, f)| \leqslant & \frac{\rho_{i}}{\pi^{3 / 2}} \sum_{i=1}^{2} \int_{\mathbb{R}^{3}} d p\left|\frac{\partial \varphi_{i}}{\partial t}+\left(\frac{p}{\sqrt{\beta_{i}}}+\bar{V}_{i}\right) \frac{\partial \varphi_{i}}{\partial x}\right| e^{-p^{2}}+ \\
& +\frac{4 \varphi_{1} \varphi_{2} d^{2} \rho_{1} \rho_{2}}{\pi^{2}} \int_{\mathbb{R}^{3}} \int_{\mathbb{R}^{3}} d q d q_{1} e^{-q^{2}-q_{1}^{2}} \Theta
\end{aligned}
$$

Домножая обе части неравенства $(32)$ на $1 /(1+|t|)$ и переходя к супремуму по всем $(t, x) \in \mathbb{R}^{4}$, имеем

$$
\begin{aligned}
\widetilde{\Delta} \leqslant \widetilde{\Delta}^{\prime}= & \sup _{(t, x) \in \mathbb{R}^{4}} \frac{1}{1+|t|}\left[\sum_{i=1}^{2} \int_{\mathbb{R}^{3}} d p\left|\frac{\partial \varphi_{i}}{\partial t}+\left(\frac{p}{\sqrt{\beta_{i}}}+\bar{V}_{i}\right) \frac{\partial \varphi_{i}}{\partial x}\right| \frac{\rho_{i}}{\pi^{3 / 2}} e^{-p^{2}}+\right. \\
& +\frac{4 \varphi_{1} \varphi_{2} d^{2} \rho_{1} \rho_{2}}{\pi^{2}} \int_{\mathbb{R}^{3}} \int_{\mathbb{R}^{3}} d q d q_{1} e^{\left.-q^{2}-q_{1}^{2} \Theta\right]}
\end{aligned}
$$

Выражение (33), как легко видеть, имеет смысл в силу выполнения условий (12)-(14). Теперь воспользуемся представлением функции $\varphi_{i}$ (12) и вычислим ее производную по переменной $t$ и пространственный градиент:

$$
\begin{aligned}
\frac{\partial \varphi_{i}}{\partial t} & =\frac{\partial \psi_{i}}{\partial t} e^{-2 \beta_{i} \bar{u}_{i} x} \\
\frac{\partial \varphi_{i}}{\partial x} & =e^{-2 \beta_{i} \bar{u}_{i} x}\left(\frac{\partial \psi_{i}}{\partial x}-2 \beta_{i} \bar{u}_{i} \psi_{i}\right) .
\end{aligned}
$$

Далее, подставляя выражения для производных (34) и используя равенство (10), из оценки (33) получим

$$
\begin{aligned}
\widetilde{\Delta}^{\prime}= & \sup _{(t, x) \in \mathbb{R}^{4}} \frac{1}{1+|t|} \sum_{i=1}^{2} \int_{\mathbb{R}^{3}} d p \mid \frac{\partial \psi_{i}}{\partial t}+\left(\frac{p}{\sqrt{\beta_{i}}}+\widehat{V}_{i}-\bar{u}_{i} t\right) \frac{\partial \psi_{i}}{\partial x}+ \\
& +2 \beta_{i} \psi_{i}\left(-\frac{1}{\sqrt{\beta_{i}}}\left(\bar{u}_{i}, p\right)-\left(\bar{u}_{i}, \widehat{V}_{i}\right)+\bar{u}_{i}^{2} t\right) \mid \frac{\rho_{0 i}}{\pi^{3 / 2}} e^{\beta_{i}\left(\widehat{V}_{i}-\bar{u}_{i} t\right)^{2}} e^{-p^{2}}+ \\
& +\sup _{(t, x) \in \mathbb{R}^{4}} \frac{1}{1+|t|} \frac{4 \psi_{1} \psi_{2} d^{2} \rho_{01} \rho_{02} e^{\beta_{1}\left(\widehat{V}_{1}-\bar{u}_{1} t\right)^{2}+\beta_{2}\left(\widehat{V}_{2}-\bar{u}_{2} t\right)^{2}}}{\pi^{2}} \times \\
& \times \int_{\mathbb{R}^{3}} \int_{\mathbb{R}^{3}} d q d q_{1} e^{-q^{2}-q_{1}^{2}} \Theta .
\end{aligned}
$$

Само существование величины $\widetilde{\Delta}^{\prime}$ вытекает из предположения об ограниченности величин (14) после умножения на функцию (13) и вида выражения (35). Далее осуществим низкотемпературный предельный переход $\left(\beta_{i} \rightarrow+\infty\right)$ в утверждении (35), возможность которого обосновывается с помощью леммы 1 работы [11] и стандарт- 
ных теорем о предельном переходе под знаком интеграла. Условия всех этих теорем легко проверяются благодаря структуре выражения (35), гладкости функций, которые входят в него, и хорошей сходимости всех интегралов.

Принимая во внимание соотношения (15), (16) и формулу (31), убеждаемся в том, что

$$
\lim _{\beta_{i} \rightarrow+\infty} \Theta=0 .
$$

Используя вновь условия (15) и (16) теоремы, получаем

$$
\lim _{\beta_{i} \rightarrow+\infty} e^{\beta_{i}\left(\widehat{V}_{i}-\bar{u}_{i} t\right)^{2}}= \begin{cases}e^{s_{i}^{2} \bar{u}_{0 i}^{2} t^{2}}, & k_{i}>\frac{1}{2}, \\ e^{s_{i}^{2}\left(\widehat{V}_{0 i}-\bar{u}_{0 i} t\right)^{2}}, & k_{i}=\frac{1}{2} .\end{cases}
$$

Кроме того, очевидно, существует предел

$$
\begin{aligned}
& \lim _{\beta_{i} \rightarrow+\infty}\left[\left(\frac{p}{\sqrt{\beta_{i}}}+\widehat{V}_{i}-\bar{u}_{i} t\right) \frac{\partial \psi_{i}}{\partial x}+2 \beta_{i} \psi_{i}\left(-\frac{1}{\sqrt{\beta_{i}}}\left(\bar{u}_{i}, p\right)-\left(\bar{u}_{i}, \widehat{V}_{i}\right)+\bar{u}_{i}^{2} t\right)\right]= \\
& = \begin{cases}2 \psi_{i}\left(t s_{i}^{2} \bar{u}_{0 i}^{2}-s_{i}\left(\bar{u}_{0 i}, p\right)\right), & k_{i}>\frac{1}{2}, \\
2 \psi_{i}\left(t s_{i}^{2} \bar{u}_{0 i}^{2}-s_{i}^{2}\left(\bar{u}_{0 i}, \widehat{V}_{0 i}\right)-s_{i}\left(\bar{u}_{0 i}, p\right)\right), & k_{i}=\frac{1}{2} .\end{cases}
\end{aligned}
$$

Таким образом, с учетом (36)-(38) получаем, что результат перехода к низкотемпературному пределу в равенстве $(35)$ при $k_{i}>1 / 2$ с последующей оценкой сверху после вычисления соответствующих интегралов и выполнения элементарных преобразований примет вид

$$
\begin{aligned}
& \lim _{\beta_{i} \rightarrow+\infty} \widetilde{\Delta}^{\prime}=\sup _{(t, x) \in \mathbb{R}^{4}} \frac{1}{1+|t|} \sum_{i=1}^{2} \int_{\mathbb{R}^{3}} d p\left|\frac{\partial \psi_{i}}{\partial t}+2 \psi_{i}\left(t s_{i}^{2} \bar{u}_{0 i}^{2}-s_{i}\left(\bar{u}_{0 i}, p\right)\right)\right| \frac{\rho_{0 i}}{\pi^{3 / 2}} e^{s_{i}^{2} \bar{u}_{0 i}^{2} t^{2}} e^{-p^{2}} \leqslant \\
& \leqslant \sup _{(t, x) \in \mathbb{R}^{4}} \frac{1}{1+|t|} \sum_{i=1}^{2} \int_{\mathbb{R}^{3}} d p\left|\frac{\partial \psi_{i}}{\partial t}+2 \psi_{i} t s_{i}^{2} \bar{u}_{0 i}^{2}\right| \frac{\rho_{0 i}}{\pi^{3 / 2}} e^{s_{i}^{2} \bar{u}_{0 i}^{2} t^{2}} e^{-p^{2}}+ \\
& +\sup _{(t, x) \in \mathbb{R}^{4}} \frac{1}{1+|t|} \sum_{i=1}^{2} \int_{\mathbb{R}^{3}} d p 2 \psi_{i} s_{i}\left|\bar{u}_{0 i}\right||p| \frac{\rho_{0 i}}{\pi^{3 / 2}} e^{s_{i}^{2} \bar{u}_{0 i}^{2} t^{2}} e^{-p^{2}}= \\
& =\sum_{i=1}^{2} \rho_{0 i} \sup _{(t, x) \in \mathbb{R}^{4}}\left\{\frac{1}{1+|t|} e^{t^{2} s_{i}^{2} \bar{u}_{0 i}^{2}}\left|\frac{\partial \psi_{i}}{\partial t}+2 \psi_{i} t s_{i}^{2} \bar{u}_{0 i}^{2}\right|\right\}+ \\
& +\frac{4}{\sqrt{\pi}} \sum_{i=1}^{2} s_{i} \rho_{0 i}\left|\bar{u}_{0 i}\right| \sup _{(t, x) \in \mathbb{R}^{4}}\left\{\frac{1}{1+|t|} e^{t^{2} s_{i}^{2} \bar{u}_{0 i}^{2}} \psi_{i}\right\}
\end{aligned}
$$

что и доказывает утверждение (19). 
В случае $k_{i}=1 / 2$, также используя результаты (36)-(38), получим

$$
\begin{aligned}
\lim _{\beta_{i} \rightarrow+\infty} \widetilde{\Delta}^{\prime}= & \sup _{(t, x) \in \mathbb{R}^{4}} \frac{1}{1+|t|} \sum_{i=1}^{2} \int_{\mathbb{R}^{3}} d p\left|\frac{\partial \psi_{i}}{\partial t}+2 \psi_{i}\left(t s_{i}^{2} \bar{u}_{0 i}^{2}-s_{i}^{2}\left(\bar{u}_{0 i}, \widehat{V}_{0 i}\right)-s_{i}\left(\bar{u}_{0 i}, p\right)\right)\right| \times \\
& \times \frac{\rho_{0 i}}{\pi^{3 / 2}} e^{s_{i}^{2}\left(\widehat{V}_{0 i}-\bar{u}_{0 i} t\right)^{2}} e^{-p^{2}} \leqslant \\
\leqslant & \sum_{i=1}^{2} \rho_{0 i} \sup _{(t, x) \in \mathbb{R}^{4}}\left\{\frac{1}{1+|t|}\left|\frac{\partial \psi_{i}}{\partial t}+2 \psi_{i} t s_{i}^{2} \bar{u}_{0 i}^{2}\right| e^{s_{i}^{2}\left(\widehat{V}_{0 i}-\bar{u}_{0 i} t\right)^{2}}\right\}+ \\
& +2 \sum_{i=1}^{2} \rho_{0 i}\left(\frac{2 s_{i}\left|\bar{u}_{0 i}\right|}{\sqrt{\pi}}+s_{i}^{2}\left(\bar{u}_{0 i}, \widehat{V}_{0 i}\right)\right) \sup _{(t, x) \in \mathbb{R}^{4}}\left\{\frac{1}{1+|t|} e^{s_{i}^{2}\left(\widehat{V}_{0 i}-\bar{u}_{0 i} t\right)^{2}} \psi_{i}\right\},
\end{aligned}
$$

а это означает истинность утверждения (20).

Теперь рассмотрим несколько следствий, в которых указаны условия, достаточные для минимизации невязки $\widetilde{\Delta}(7)$.

СлеДСТвиЕ 1. Пусть функиии $\psi_{i}$ при $k_{i}>1 / 2$ имеют вид

$$
\psi_{i}(t, x)=C_{i}(x) e^{-s_{i}^{2} \bar{u}_{0 i}^{2} t^{2}}
$$

где $C_{i}(x)$ - произвольные положительные, гладкие, ограниченные вместе со своими производными на $\mathbb{R}^{3}$ функции. Тогда невязка $\widetilde{\Delta}$ является произвольно малой при достаточно малых значениях $s_{1}, s_{2}, T_{1}, T_{2}$, m.е. для любого $\varepsilon>0$ найдутся $\delta>0$ и $\beta_{0}>0$ такие, что для всех $s_{1}, s_{2}$, удовлетворяющих неравенству $0<s_{1}, s_{2}<\delta$, и всех $\beta_{1}, \beta_{2}>\beta_{0}$ выполнено неравенство $\widetilde{\Delta}<\varepsilon$.

ДокАЗАТЕЛьство. Для начала проверим, что функции $\psi_{i}$ вида (39) при $k_{i}>1 / 2$ и достаточно малых $T_{1}, T_{2}>0$ обеспечивают ограниченность функций (14), умноженных на функцию (13). Проверим это для одной из функций (14) (для остальных это проверяется аналогично):

$$
\begin{aligned}
t \psi_{i} e^{\beta_{i}\left(\widehat{V}_{i}-\bar{u}_{i} t\right)^{2}} \frac{1}{1+|t|} & =\frac{t}{1+|t|} C_{i}(x) e^{\beta_{i}\left(\widehat{V}_{i}-\bar{u}_{i} t\right)^{2}-s_{i}^{2} \bar{u}_{0 i}^{2} t^{2}}= \\
& =\frac{t}{1+|t|} C_{i}(x) \exp \left[\beta_{i}\left(\frac{s_{i} \widehat{V}_{0 i}}{\beta_{i}^{k_{i}}}-\frac{s_{i} \bar{u}_{0 i}}{\sqrt{\beta_{i}}} t\right)^{2}-s_{i}^{2} \bar{u}_{0 i}^{2} t^{2}\right]= \\
& =\frac{t}{1+|t|} C_{i}(x) \exp \left[s_{i}^{2} \widehat{V}_{0 i}^{2} \beta_{i}^{1-2 k_{i}}-2 s_{i}^{2}\left(\bar{u}_{0 i}, \widehat{V}_{0 i}\right) t \beta_{i}^{1-k_{i}}\right] .
\end{aligned}
$$

Вычислим производную функции $\psi_{i}$ по переменной $t$, которая входит в равенство (19):

$$
\frac{\partial \psi_{i}}{\partial t}=C_{i}(x) e^{-s_{i}^{2} \bar{u}_{0 i}^{2} t^{2}}\left(-2 s_{i}^{2} \bar{u}_{0 i}^{2} t\right)=-2 \psi_{i} s_{i}^{2} \bar{u}_{0 i}^{2} t
$$

Подставляя выражение для производной (41) в равенство (19) и используя достаточную малость величин $s_{1}, s_{2}, T_{1}, T_{2}$, получаем утверждение следствия 1. 
СЛЕДСТВИЕ 2. Пусть в случае $k_{i}=1 / 2$ остаются верны все условия следствия 1 и выполняется еще одно условие:

$$
\bar{u}_{i} \perp \widehat{V}_{i}
$$

Тогда утверждение следствия 1 остается в силе.

ДокАЗАТЕЛЬСтво. Снова проверим ограниченность одной из величин (14), умноженной на функцию (13), для функции $\psi_{i}$ вида (39):

$$
t \psi_{i} e^{\beta_{i}\left(\widehat{V}_{i}-\bar{u}_{i} t\right)^{2}} \frac{1}{1+|t|}=\frac{t}{1+|t|} C_{i}(x) e^{s_{i}^{2} \widehat{V}_{0 i}^{2} \beta_{i}^{1-2 k_{i}}} .
$$

Далее, используя производную (41) и малость величин $s_{1}, s_{2}, T_{1}, T_{2}$, а также условие (42), помогающее обратить в нуль второе слагаемое в правой части равенства (20), вновь получаем утверждение следствия 1.

Теорема 2. Пусть имеет место следующее представление:

$$
\varphi_{i}(t, x)=C_{i}\left(x+\bar{u}_{i} \frac{\left(\widehat{V}_{i}-\bar{u}_{i} t\right)^{2}}{2 \bar{u}_{i}^{2}}\right)
$$

где $C_{i}$ - неотрицательные, финитные или достаточно быстро убывающие на бесконечности гладкие функиии. Пусть также имеют место соотношения

$$
\begin{gathered}
\bar{u}_{i}=\bar{u}_{0 i} \beta_{i}^{-n_{i}} \\
\widehat{V}_{i}=\widehat{V}_{0 i} \beta_{i}^{-k_{i}}, \\
n_{i} \geqslant 1 ; \quad k_{i} \geqslant \frac{1}{2}, \quad k_{i} \geqslant \frac{n_{i}}{2} .
\end{gathered}
$$

Тогда верно утверждение

$$
\lim _{\beta_{i} \rightarrow+\infty} \widetilde{\Delta}=0
$$

ДокАзАтЕльство. Легко показать, что функции

$$
t \varphi_{i} \rho_{i}(t, x), \quad \frac{\partial \varphi_{i}}{\partial t} \rho_{i}(t, x), \quad\left|\frac{\partial \varphi_{i}}{\partial x}\right| \rho_{i}(t, x), \quad t\left(\bar{u}_{i}, \frac{\partial \varphi_{i}}{\partial x}\right) \rho_{i}(t, x)
$$

после умножения на $1 /(1+|t|)$ ограниченны на пространстве $\mathbb{R}^{4}$. Далее, в силу предположения, что $\varphi_{i}$ - гладкая функция, получаем, что такое же "ограничение с весом" имеет место и для функций $\sqrt{|t|} \varphi_{i} \rho_{i}(t, x),|t| \varphi_{1} \varphi_{2} \rho_{1}(t, x) \rho_{2}(t, x)$, а также для $\varphi_{1} \varphi_{2} \rho_{1}(t, x) \rho_{2}(t, x)$. Значит, корректно определена оценка $(18)$.

Очевидно, что остается верным неравенство (33), а правая часть выражения (10) с учетом предположений $(44),(45)$ имеет вид

$$
\rho_{0 i} \exp \left[\beta_{i}\left(\left(\frac{\widehat{V}_{0 i}}{\beta_{i}^{k_{i}}}-\frac{\bar{u}_{0 i}}{\beta_{i}^{n_{i}}} t\right)^{2}+\frac{2 \bar{u}_{0 i} x}{\beta_{i}^{n_{i}}}\right)\right] .
$$


Найдем производные по $t$ и $x$ функции $\varphi_{i}$, используя ее представление (43):

$$
\begin{aligned}
\frac{\partial \varphi_{i}}{\partial x} & =C_{i}^{\prime}\left(x+\bar{u}_{i} \frac{\left(\widehat{V}_{i}-\bar{u}_{i} t\right)^{2}}{2 \bar{u}_{i}^{2}}\right) \\
\frac{\partial \varphi_{i}}{\partial t} & =\frac{t \bar{u}_{i}^{2}-\left(\bar{u}_{i}, \widehat{V}_{i}\right)}{\bar{u}_{i}^{2}}\left(\bar{u}_{i}, C_{i}^{\prime}\left(x+\frac{\bar{u}_{i}\left(\widehat{V}_{i}-\bar{u}_{i} t\right)^{2}}{2 \bar{u}_{i}^{2}}\right)\right) .
\end{aligned}
$$

Теперь перейдем к низкотемпературному пределу в правой части (33) (обоснование возможности такого перехода аналогично сделанному в доказательстве теоремы 1).

Таким образом, используя выражение (48) и условие (46), можно утверждать, чTо

$$
\lim _{\beta_{i} \rightarrow+\infty} \rho_{i}=\rho_{0 i} \eta_{i}(x)
$$

где введено обозначение

$$
\eta_{i}(x)= \begin{cases}1, & n_{i}>1, k_{i}>\frac{1}{2}, \\ e^{2 \bar{u}_{0 i} x}, & n_{i}=1, k_{i}>\frac{1}{2} \\ e^{\widehat{V}_{0 i}^{2}+2 \bar{u}_{0 i} x}, & n_{i}=1, k_{i}=\frac{1}{2} .\end{cases}
$$

Принимая во внимание условия (44), (45), перейдем также к пределу в самом выражении (43):

$$
\lim _{\beta_{i} \rightarrow+\infty} \varphi_{i}=C\left(x+a_{i}\right)
$$

где

$$
a_{i}= \begin{cases}0, & k_{i}>\frac{n_{i}}{2}, \\ \frac{\bar{u}_{0 i} \widehat{V}_{0 i}^{2}}{2 \bar{u}_{0 i}^{2}}, & k_{i}=\frac{n_{i}}{2} .\end{cases}
$$

Теперь вычислим пределы производных функции $\varphi_{i}$, пользуясь их явным видом (49), а также условиями (44), (45) теоремы:

$$
\begin{aligned}
& \lim _{\beta_{i} \rightarrow+\infty} \frac{\partial \varphi_{i}}{\partial t}=0, \\
& \lim _{\beta_{i} \rightarrow+\infty} \frac{\partial \varphi_{i}}{\partial x}=C_{i}^{\prime}\left(x+a_{i}\right) .
\end{aligned}
$$

В случае функции вида (43) очевидно выполнение равенства (36). Тогда, используя ранее найденные значения (50)-(54), можно утверждать, что

$$
\lim _{\beta_{i} \rightarrow+\infty} \Delta^{\prime}=0
$$

а значит, справедливо и утверждение (47). 
ТЕОрема 3. Пусть функиии $\varphi_{i}$ в распределении (8) имеют вид

$$
\varphi_{i}(t, x)=\psi_{i}(t, x) e^{-\beta_{i}\left(\left(\widehat{V}_{i}-\bar{u}_{i} t\right)^{2}+2 \bar{u}_{i} x\right)},
$$

где $\psi_{i}(t, x)$ таковы, что выражения

$$
t \psi_{1} \psi_{2}, \quad \frac{\partial \psi_{i}}{\partial t}, \quad t \psi_{i}, \quad\left|\frac{\partial \psi_{i}}{\partial x}\right|, \quad t\left(\bar{u}_{i}, \frac{\partial \psi_{i}}{\partial x}\right)
$$

умноженные на $1 /(1+|t|)$, ограниченны на $\mathbb{R}^{4}$. Пусть также выполняется представление (44) для $n_{i}>1 / 2$.

Тогда верна оценка (18), причем

$$
\begin{aligned}
\lim _{\beta_{i} \rightarrow+\infty} \Delta^{\prime}= & \sum_{i=1}^{2} \rho_{0 i} \sup _{(t, x) \in \mathbb{R}^{4}} \frac{1}{1+|t|}\left|\frac{\partial \psi_{i}}{\partial t}+\widehat{V}_{i} \frac{\partial \psi_{i}}{\partial x}\right|+ \\
& +4 \pi d^{2} \rho_{01} \rho_{02}\left|\widehat{V}_{1}-\widehat{V}_{2}\right| \sup _{(t, x) \in \mathbb{R}^{4}} \frac{1}{1+|t|}\left(\psi_{1} \psi_{2}\right) .
\end{aligned}
$$

ДокАзАтЕЛЬство. Как и выше, остается верным неравенство (33). Найдем производные функции $\varphi_{i}(56)$ по времени $t$ и по пространственной координате $x$ :

$$
\begin{aligned}
\frac{\partial \varphi_{i}}{\partial t} & =\rho_{0 i} \rho_{i}^{-1}(t, x)\left[\frac{\partial \psi_{i}}{\partial t}+2 \beta_{i} \psi_{i}\left(\left(\widehat{V}_{i}, \bar{u}_{i}\right)-t \bar{u}_{i}^{2}\right)\right], \\
\frac{\partial \varphi_{i}}{\partial x} & =\rho_{0 i} \rho_{i}^{-1}(t, x)\left[\frac{\partial \psi_{i}}{\partial x}-2 \beta_{i} \psi_{i} \bar{u}_{i}\right] .
\end{aligned}
$$

Подставим найденные производные в неравенство (33):

$$
\begin{aligned}
\widetilde{\Delta}^{\prime}=\pi^{-3 / 2} \sup _{(t, x) \in \mathbb{R}^{4}} \frac{1}{1+|t|} \sum_{i=1}^{2} \int_{\mathbb{R}^{3}} d p \mid \rho_{0 i} \rho_{i}^{-1}(t, x)\left[\frac{\partial \psi_{i}}{\partial t}+2 \beta_{i} \psi_{i}\left(\left(\widehat{V}_{i}, \bar{u}_{i}\right)-t \bar{u}_{i}^{2}\right)\right]+ \\
+\left(\frac{p}{\sqrt{\beta_{i}}}+\widehat{V}_{i}-\bar{u}_{i} t\right) \rho_{0 i} \rho_{i}^{-1}(t, x)\left(\frac{\partial \psi_{i}}{\partial x}-2 \beta_{i} \psi_{i} \bar{u}_{i}\right) \mid \rho_{i} e^{-p^{2}}+ \\
+\frac{4 d^{2} \rho_{01} \rho_{02}}{\pi^{2}} \sup _{(t, x) \in \mathbb{R}^{4}} \frac{1}{1+|t|} \psi_{1} \psi_{2} \int_{\mathbb{R}^{3}} d q \int_{\mathbb{R}^{3}} d q_{1} e^{-q^{2}-q_{1}^{2}} \Theta
\end{aligned}
$$

Преобразуем полученное выражение:

$$
\begin{aligned}
\widetilde{\Delta}^{\prime} & =\pi^{-3 / 2} \sup _{(t, x) \in \mathbb{R}^{4}} \frac{1}{1+|t|} \sum_{i=1}^{2} \int_{\mathbb{R}^{3}} d p \mid \frac{\partial \psi_{i}}{\partial t}+\left(\frac{p}{\sqrt{\beta_{i}}}+\widehat{V}_{i}-\bar{u}_{i} t\right) \frac{\partial \psi_{i}}{\partial x}- \\
& -2 \sqrt{\beta_{i}} \psi_{i}\left(\bar{u}_{i}, p\right) \mid \rho_{0 i} e^{-p^{2}}+\frac{4 d^{2} \rho_{01} \rho_{02}}{\pi^{2}} \sup _{(t, x) \in \mathbb{R}^{4}} \frac{1}{1+|t|} \psi_{1} \psi_{2} \int_{\mathbb{R}^{3}} d q \int_{\mathbb{R}^{3}} d q_{1} e^{-q^{2}-q_{1}^{2} \Theta .}
\end{aligned}
$$


Далее, снова выполняя предельный переход в равенстве (60) и принимая во внимание, что низкотемпературный предел величины (31) с учетом условия (44) теоремы имеет вид

$$
\lim _{\beta_{i} \rightarrow+\infty} \Theta=\left|\widehat{V}_{1}-\widehat{V}_{2}\right|
$$

видим, что соответствующий предел величины $\widetilde{\Delta}^{\prime}$, описываемой равенством $(60)$ при выполнении (44) и (61), будет иметь вид (58).

СлеДСТВИЕ 3. Пусть функции $\psi_{i}$ в (56) имеют вид

$$
\psi_{i}=C_{i}\left(x-\widehat{V}_{i} t\right)
$$

или

$$
\psi_{i}=C_{i}\left(\left[x \times \widehat{V}_{i}\right]\right),
$$

где $C_{i}$ - неотрицательные, гладкие, финитные функции.

Тогда справедливы следующие утверждения:

1) если выполняется равенство

$$
\operatorname{supp} C_{1} \cap \operatorname{supp} C_{2}=\varnothing
$$

или

$$
\widehat{V}_{1}=\widehat{V}_{2}
$$

то верно утверждение (47);

2) при произвольных $C_{1}, C_{2}, \widehat{V}_{1}, \widehat{V}_{2}$ имеем

$$
\lim _{d \rightarrow 0} \lim _{\beta_{i} \rightarrow+\infty} \Delta^{\prime}=0 .
$$

ДокАЗАтельство. Указанные функции $\psi_{i}$ удовлетворяют условиям теоремы 3. Очевидно, что в случае (62)

$$
\frac{\partial \psi_{i}}{\partial t}=-\left(\widehat{V}_{i}, C_{i}^{\prime}\right), \quad \frac{\partial \psi_{i}}{\partial x}=C_{i}^{\prime}
$$

а в случае (63)

$$
\frac{\partial \psi_{i}}{\partial t}=0, \quad \frac{\partial \psi_{i}}{\partial x}=\left[\widehat{V}_{i} \times C_{i}^{\prime}\right]
$$

Найденные производные (67), (68) обращают в нуль первое слагаемое в правой части равенства (58), а дополнительные условия (64), (65) или условие $d \rightarrow 0$ обращают в нуль второе слагаемое в правой части равенства (58). Таким образом, выполняются утверждения (47) или (66) соответственно.

ТЕОрема 4. Предположим, что функиии $\varphi_{i}(t, x)$ имеют вид

$$
\varphi_{i}(t, x)=\psi_{i}(t, x) e^{-\beta_{i}\left(\widehat{V}_{i}-\bar{u}_{i} t\right)^{2}},
$$

причем выражения

$$
\begin{aligned}
t \psi_{1} \psi_{2} e^{2 \beta_{1} \bar{u}_{1} x+2 \beta_{2} \bar{u}_{2} x}, & \frac{\partial \psi_{i}}{\partial t} e^{2 \beta_{i} \bar{u}_{i} x}, \quad t \psi_{i} e^{2 \beta_{i} \bar{u}_{i} x}, \\
\left|\frac{\partial \psi_{i}}{\partial x}\right| e^{2 \beta_{i} \bar{u}_{i} x}, & t\left(\bar{u}_{i}, \frac{\partial \psi_{i}}{\partial x}\right) e^{2 \beta_{i} \bar{u}_{i} x}
\end{aligned}
$$

ограниченны с весом $1 /(1+|t|)$, а условие (44) верно при $n_{i} \geqslant 1$. 
Тогда имеет место оценка (18), причем в случае $n_{i}>1$ выполняется утверждение (58), а при $n_{i}=1$ низкотемпературный предел величины $\widetilde{\Delta}^{\prime}$ имеет вид

$$
\begin{aligned}
\lim _{\beta_{i} \rightarrow+\infty} \Delta^{\prime}=\sum_{i=1}^{2} \rho_{0 i} \sup _{(t, x) \in \mathbb{R}^{4}} \frac{1}{1+|t|}\left|\mu_{i}(x)\left(\frac{\partial \psi_{i}}{\partial t}+\widehat{V}_{i} \frac{\partial \psi_{i}}{\partial x}\right)\right|+ \\
+4 \pi d^{2} \rho_{01} \rho_{02}\left|\widehat{V}_{1}-\widehat{V}_{2}\right| \sup _{(t, x) \in \mathbb{R}^{4}} \frac{1}{1+|t|}\left[\mu_{1}(x) \mu_{2}(x) \psi_{1}(t, x) \psi_{2}(t, x)\right]+ \\
+2 \sum_{i=1}^{2} \rho_{0 i}\left|\left(\bar{u}_{0 i}, \widehat{V}_{i}\right)\right| \sup _{(t, x) \in \mathbb{R}^{4}} \frac{1}{1+|t|}\left[\mu_{i}(x) \psi_{i}(t, x)\right]
\end{aligned}
$$

əəe

$$
\mu_{i}(x)=e^{2 \bar{u}_{0 i} x}
$$

ДокАЗАтЕЛЬство. Используем неравенство (33), которое, очевидно, сохраняет силу. Для этого сначала найдем производные функции $\varphi_{i}$ вида (69) по переменным $t$ и $x$ :

$$
\begin{aligned}
\frac{\partial \varphi_{i}}{\partial x} & =e^{-\beta_{i}\left(\widehat{V}_{i}-\bar{u}_{i} t\right)^{2}} \frac{\partial \psi_{i}}{\partial x} \\
\frac{\partial \varphi_{i}}{\partial t} & =e^{-\beta_{i}\left(\widehat{V}_{i}-\bar{u}_{i} t\right)^{2}}\left[\frac{\partial \psi_{i}}{\partial t}+2 \beta_{i} \psi_{i}\left(\left(\bar{u}_{i}, \widehat{V}_{i}\right)-t \bar{u}_{i}^{2}\right)\right]
\end{aligned}
$$

Найденные производные подставим в неравенство (33) и получим следующее выражение для $\widetilde{\Delta}^{\prime}$ :

$$
\begin{aligned}
\widetilde{\Delta}^{\prime}= & \sup _{(t, x) \in \mathbb{R}^{4}} \frac{1}{1+|t|} \sum_{i=1}^{2} \int_{\mathbb{R}^{3}} d p \mid \frac{\partial \psi_{i}}{\partial t}+2 \beta_{i} \psi_{i}\left(\left(\bar{u}_{i}, \widehat{V}_{i}\right)-t \bar{u}_{i}^{2}\right)+ \\
& +\left(\frac{p}{\sqrt{\beta_{i}}}+\widehat{V}_{i}-\bar{u}_{i} t\right) \frac{\partial \psi_{i}}{\partial x} \mid \frac{\rho_{0 i} e^{2 \beta_{i} \bar{u}_{i} x}}{\pi^{3 / 2}} e^{-p^{2}}+ \\
& +\sup _{(t, x) \in \mathbb{R}^{4}} \frac{1}{1+|t|} \frac{4 \rho_{01} \rho_{02} \psi_{1} \psi_{2} d^{2} e^{2 \beta_{1} \bar{u}_{1} x+2 \beta_{2} \bar{u}_{2} x}}{\pi^{2}} \int_{\mathbb{R}^{3}} d q \int_{\mathbb{R}^{3}} d q_{1} e^{-q^{2}-q_{1}^{2}} \Theta,
\end{aligned}
$$

что не превосходит выражения

$$
\begin{aligned}
& \sum_{i=1}^{2} \frac{\rho_{0 i}}{\pi^{3 / 2}} \sup _{(t, x) \in \mathbb{R}^{4}} \frac{1}{1+|t|} \int_{\mathbb{R}^{3}} d p\left|\frac{\partial \psi_{i}}{\partial t}+\left(\frac{p}{\sqrt{\beta_{i}}}+\widehat{V}_{i}-\bar{u}_{i} t\right) \frac{\partial \psi_{i}}{\partial x}\right| e^{2 \beta_{i} \bar{u}_{i} x} e^{-p^{2}}+ \\
& \quad+\sup _{(t, x) \in \mathbb{R}^{4}} \frac{1}{1+|t|} \frac{4 \rho_{01} \rho_{02} \psi_{1} \psi_{2} d^{2} e^{2 x\left(\beta_{1} \bar{u}_{1}+\beta_{2} \bar{u}_{2}\right)}}{\pi^{2}} \int_{\mathbb{R}^{3}} d q \int_{\mathbb{R}^{3}} d q_{1} e^{-q^{2}-q_{1}^{2}} \Theta+ \\
& \quad+2 \sum_{i=1}^{2} \frac{\rho_{0 i}}{\pi^{3 / 2}} \sup _{(t, x) \in \mathbb{R}^{4}} \frac{1}{1+|t|} \int_{\mathbb{R}^{3}} d p \beta_{i} \psi_{i}\left|\left(\left(\bar{u}_{i}, \widehat{V}_{i}\right)-t \bar{u}_{i}^{2}\right)\right| e^{2 \beta_{i} \bar{u}_{i} x} e^{-p^{2}}
\end{aligned}
$$

Далее, переходя к низкотемпературному пределу в последнем выражении и используя условие (44), получаем формулу (58) при $n_{i}>1$, а в случае $n_{i}=1$ имеем утверждение $(71)$ с функциями $\mu_{i}(x)$ вида $(72)$. 
В теореме 4 получен предел величины $\widetilde{\Delta}^{\prime}$ из оценки (18), что позволяет сформулировать еще одно достаточное условие минимизации невязки (7).

СлЕДСТВИЕ 4. Пусть выполняются условие (44) при $n_{i} \geqslant 1$ и условие (42) следствия 2, а функиии $\psi_{i}$ имеют вид (62) или (63). Тогда справедливы утверждения (47) и (66).

ДокАзАтельство. Сначала проверим, что данные функции при выполнении условий этого следствия удовлетворяют требованию теоремы 4. Выражение $t \psi_{1} \psi_{2} e^{2 \beta_{1} \bar{u}_{1} x+2 \beta_{2} \bar{u}_{2} x}$ равно нулю в силу первого утверждения следствия 3 , а в случае $\widehat{V}_{1}=\widehat{V}_{2}$ сделаем замену $y=x-\widehat{V}_{i} t$ и получим выражение $t C_{1}(y) C_{2}(y) e^{2 y\left(\beta_{1} \bar{u}_{1}+\beta_{2} \bar{u}_{2}\right)}$, которое, учитывая финитность функции $C_{i}(y)$, после умножения на $1 /(1+|t|)$ будет ограниченно.

Если же функции $\psi_{i}$ взять в виде $(63)$ и снова предполагать, что $\widehat{V}_{1}=\widehat{V}_{2}$, то после разложения вектора $x$ по ортогональному базису $\bar{u}_{i}, \widehat{V}_{i},\left[\bar{u}_{i} \times \widehat{V}_{i}\right]$ (вследствие (42)) функция $\psi_{i}=C_{i}\left(\left[x \times \widehat{V}_{i}\right]\right)$ благодаря специфике своего аргумента обращается в нуль в тех направлениях в пространстве $\mathbb{R}^{3}$, по которым соответствующие экспоненты в условиях теоремы 4 возрастают.

Наконец, пользуясь утверждением теоремы 4, видим, что все слагаемые в (71) обращаются в нуль в силу условий данного следствия.

\section{3. ОБСУЖДЕНИЕ РЕЗУЛЬТАТОВ}

Найденные в следствии 1 решения $\psi_{i}(t, x)$ вида $(39)$ ранее для изучаемой здесь модели не встречались. Их особенностью является очень быстрое (быстрее любой степени экспоненты) убывание по времени, т.е. при $t \rightarrow \pm \infty$, а это и означает, что они являются “быстро релаксирующими решениями”. Подобные решения стали возможны только благодаря рассмотрению невязки с весом вида (7) в сочетании с условием бесконечной малости множителей $s_{i}$.

В следствиях 1, 2 описаны разные варианты поведения параметров, которые входят в уравнение Бриана-Пиддака и в само бимодальное распределение $f$, однако они приводят к одним и тем же результатам, а именно к бесконечной малости величины $\widetilde{\Delta}$. Например, если векторы $\widehat{V}_{i}$ стремятся к нулю быстрее (случай $k_{i}>1 / 2$ ), то их направление в пространстве остается произвольным; если же скорость их убывания меньше, что соответствует $k_{i}=1 / 2$, то необходимо привлечение дополнительного условия (42), которое обеспечивает ограниченность по $t$ соответствующих выражений (14) с весом (13) (см. также второе слагаемое в (20)), а значит, конечность самой невязки (7) и ее предела.

Интересно, что при отказе от предположения (17) или при изменении степени знаменателя в (15), как можно убедиться, результаты предельных переходов по $\beta_{i}$ становятся просто бесконечными или тривиальными.

Также отметим, что в теореме 1 параметр $d$, который входит в формулу (3), остается фиксированным, в то время как при получении других результатов рассматривается и предельный переход $d \rightarrow 0$. Физически это означает, что "быстро релаксирующие решения" возможны и для больцмановского газа, т. е. при конечных значениях числа Кнудсена [9], а не только в случае стремления числа Кнудсена к бесконечности (очень сильно разреженный газ). 


\section{Список литературы}

[1] G. H. Bryan, Rep. Brit. Assoc. Adv. Sci., 64 (1894), 102-106.

[2] F. B. Pidduck, Proc. R. Soc. Lond. A, 101:708 (1922), 101-112.

[3] С. Чепмен, Т. Каулинг, Математическая теория неоднородных газов, ИЛ, М., 1960.

[4] C. Cercignani, M. Lampic, J. Statist. Phys., 53:3-4 (1988), 655-672.

[5] V.D. Gordevskyy, Math. Meth. Appl. Sci., 23:13 (2000), 1121-1137.

[6] В. Д. Гордевский, А. А. Гукалов, Укр. матем. журн., 63:5 (2011), 629-639.

[7] В. Д. Гордевский, Докл. НАН Украины. Сер. Матем., естествознание и техн. науки, 11 (2009), 13-16.

[8] V.D. Gordevskyy, N.V. Andriyasheva, ЖКурн. матем. физ., анал., геом., 1:5 (2009), 38-53.

[9] К. Черчиньяни, Теория и приложения уравнения Больимана, Мир, М., 1978.

[10] В. Д. Гордевский, А. А. Гукалов, Вестн. Харък. ун-та. Сер. Матем., прикл. матем. и механ., 990:64 (2011), 27-41.

[11] В. Д. Гордевский, ТМФ, 126:2 (2001), 283-300.

Поступила в редакцию 13.12.2012 